\title{
CONNECTIVITY OF AN INTERVAL
}

\author{
MARK MANDELKER ${ }^{1}$
}

\begin{abstract}
Constructivizations are given for the classical theorem that an interval is connected in the sense that it cannot be the union of two nonvoid disjoint open subsets, and also for the theorem that an open set is the union of disjoint open intervals.
\end{abstract}

1. Connectivity. To find numerical meaning in the classical theorem on the connectivity of an interval, one must give an affirmative cast to the negativistic concept of the impossibility of certain partitions. The classical result states that it is impossible for open subsets of an interval to simultaneously (1) cover the interval, (2) be disjoint, and (3) be nonvoid. Three constructivizations result, each showing that under the assumption of two of the three conditions, the third is precluded. In each case, an affirmative form of the negation of the precluded condition will be proved.

For the first result, a fundamental lemma on located and totally bounded sets is needed; it is also used in $\$ 2$. The terminology and basic results of Bishop's treatise [B] will be used. For example, a located set $G$ is one from which the distance of any point on the line may be measured; its metric complement $-G$ consists of all points at a positive distance from $G$.

Lemma. Let $G$ be any located subset of the line. If the point $a$ is in $-G$, and $a<b$, then $H \equiv\{x \in[a, b]:[a, x] \subseteq-G\}$ is totally bounded.

Proof. We may assume $a=0$ and $b=1$. Let $\epsilon>0$ and choose $m$ so that $1 / m<\rho(0, G)$ and $3 / m<\epsilon$. For each $i, 0 \leqslant i \leqslant m$, choose $\sigma_{i}=0$ or 1 , so that $\sigma_{0}=1$ and

$$
\begin{aligned}
& \rho(i / m, G)>1 / m \text { when } \sigma_{i}=1, \\
& \rho(i / m, G)<2 / m \text { when } \sigma_{i}=0 .
\end{aligned}
$$

Let $n$ be the largest integer such that $\sigma_{i}=1$ for $0 \leqslant i \leqslant n$. To show that $A \equiv\{0,1 / m, \ldots, n / m\}$ is an $\epsilon$ approximation to $H$, we first show that $[0, n / m] \subseteq-G$. Let $0 \leqslant x \leqslant n / m$. Choose $i \leqslant n$ so that $|x-i / m| \leqslant 1 / m$. Then $\rho(x, G)>\rho(i / m, G)-|x-i / m|>0$, and thus $x$ is in $-G$. Hence $A$ is contained in $H$. Now let $y$ be any point of $H$ and suppose $y>(n+3) / m$. Since $y \leqslant 1$, we have $n<m$; thus $\sigma_{n+1}=0$ and $\rho((n+1) / m, G)<2 / m$. Choose $z$ in $G$ with $|(n+1) / m-z|<2 / m$; thus $z<(n+3) / m<y$ and $z$ is in $-G$, a contradiction. Hence $y \leqslant(n+3) / m$. Choose $j \leqslant n+3$ such that $|y-j / m| \leqslant 1 / m$. If $j \leqslant n$, then $j / m$ is in $A$ with $|y-j / m|<\epsilon$. If $j>n$, then $y \geqslant n / m$ so $|y-n / m| \leqslant 3 / m<\epsilon$, with $n / m$ in $A$. Thus $A$ is an $\epsilon$ ap proximation to $H$.

Received by the editors January 6, 1975.

AMS (MOS) subject classifications (1970). Primary 26A03.

Key words and phrases. Interval, connected, constructive.

${ }^{1}$ This work was supported in part by NSF Grant GP-28110. 
We shall say that two sets are disjoint if their intersection is void.

THEOREM 1. If $U$ and $V$ are nonvoid, disjoint, located, open subsets of a proper compact interval I, then these subsets do not cover the interval, in the sense that a point of I may be constructed that has a positive distance from every point of $U$ and from every point of $V$.

Proof. Choose $a \in U$ and $b \in V$; we may assume $a<b$. It follows from the lemma that $H \equiv\{x \in I:[a, x] \subseteq-V\}$ is totally bounded, and thus has a supremum $z$; clearly $a<z<b$. Let $y \in U$ and choose $\epsilon>0$ so that $(y-$ $2 \epsilon, y+2 \epsilon) \cap I \subseteq U$ and $[z-\epsilon, z+\epsilon] \subseteq I$. Suppose $|z-y|<\epsilon$; then $[z-$ $\epsilon, z+\epsilon] \subseteq-V$, so that $z+\epsilon \in H$, contradicting the definition of $z$. Hence $|z-y| \geqslant \epsilon$. Now let $y \in V$ and choose $\delta>0$ so that $(y-\delta, y+\delta) \cap I$ $\subseteq V$. Suppose $|z-y|<\delta$; then $y-\delta<z$ and there is $x \in H$ with $y-\delta$ $<x \leqslant z<y+\delta$. Hence $x \in V$, contradicting the choice of $x$ in $H$; thus $|z-y| \geqslant \delta$.

THEOREM 2. If a compact interval is the union of two nonvoid open subsets, then these subsets have a common point.

Proof. Let $I=[a, d]$ be the union of nonvoid open subsets $U$ and $V$, with $a$ in $U$. Choose any point $b$ in $V$. Put $a_{1} \equiv a, b_{1} \equiv b$, and let $c_{1}$ be the midpoint of $a_{1}$ and $b_{1}$. If $c_{1}$ is in $U$, put $a_{2} \equiv c_{1}, b_{2} \equiv b_{1}$; while if $c_{1}$ is in $V$, put $a_{2} \equiv a_{1}, b_{2} \equiv c_{1}$. Proceeding in this way, we construct a sequence of nested intervals, as in Cantor's diagonal proof [B, p. 25]. Both sequences of endpoints, $\left\{a_{n}\right\}$ and $\left\{b_{n}\right\}$, converge to the same point $c$. Since $c$ is in $I$, it is either in $U$ or in $V$; we may assume it is in $U$. Since $U$ is open, there exists $\delta>0$ so that $(c-\delta, c+\delta) \cap I \subseteq U$. Since $b_{n} \rightarrow c$, we may choose $k$ so that $b_{k} \in(c-\delta, c+\delta)$. It follows that $b_{k}$ lies in both $U$ and $V$. (It is left for the reader to give an example in which $c$ is not a common point.)

Under the hypothesis that an interval is the union of two subsets, there is given a computational procedure for determining in which of these subsets any given point of the interval lies. Thus the proof below predicts that if this calculation is carried out for any one point, then the result will be the same for all other points of the interval.

THEOREM 3. If a compact interval is the union of two disjoint open subsets, then one of these subsets is the entire interval (and the other void).

Proof. Let $I=[a, b]$ be the union of disjoint open subsets $U$ and $V$, with $a \in U$. Let $x \in I$. If $x \in V$, then $U$ and $V$ are nonvoid open subsets that cover the interval, so by Theorem 2 they have a common point, which by hypothesis is contradictory; hence $x \in U$. This shows that $U=I$.

2. Open sets and unions of disjoint open intervals. For this constructivization, we require that the given open set be colocated, i.e., it is the metric complement of a located set. With this restriction, we obtain the result in the classical form, for a bounded open set, without, however, a procedure for indexing the intervals into a countable sequence. A family $\left\{A_{i}\right\}_{i \in I}$ of sets will be called disjoint if whenever two of them have a common point, then they are equal. 
THEOREM 4. If $U$ is any bounded colocated open set on the line, then $U$ is the union of a family $\left\{I_{a}\right\}_{a \in U}$ of disjoint open intervals.

Proof. There are bounds $b$ and $c$, and a located set $G$ such that $U=\{x \in$ $[b, c]: x \in-G\}$. Clearly $b$ and $c$ are in $G$. Let $a$ be any point of $U$; then $b<a<c$. By the lemma, $J_{a} \equiv\{x \in[b, a]:[x, a] \subseteq U\}$ is totally bounded, and thus has an infimum $a_{1}$. Clearly $\left(a_{1}, a\right] \subseteq U, a_{1}<a$, and $a_{1} \in G$. Similarly, $K_{a} \equiv\{x \in[a, c]:[a, x] \subseteq U\}$ is totally bounded and has a supremum $a_{2}>a$, with $a_{2} \in G$ and $\left[a, a_{2}\right) \subseteq U$. Thus $I_{a} \equiv\left(a_{1}, a_{2}\right)$ is contained in $U$ and contains $a$. This shows that $U$ is the union of the intervals $\left\{I_{a}\right\}_{a \in U}$. Let $a, b \in U$ and $x \in I_{a} \cap I_{b}$; then $a_{1}<x<a_{2}$. Since $a_{1}, a_{2} \in G$, we have $I_{x}=I_{a}$, and similarly $I_{x}=I_{b}$. Hence $I_{a}=I_{b}$; this shows that the intervals $\left\{I_{a}\right\}_{a \in U}$ are disjoint.

\section{REFERENCE}

[B] E. Bishop, Foundations of constructive analysis, McGraw-Hill, New York, 1967. MR 36 \#4930.

Department of Mathematics, New Mexico State University, Las Cruces, New Mexico 88003

Current address: 2315 Derby Street, Berkeley, California 94705 